\title{
Pre-Treated Effect of Friction Stir Processing of Al Alloy 5052 on Vibration Fracture Behavior under Resonant Vibration
}

\author{
Kuo-Tsung Huang*1, Truan-Sheng Lui*2 and Li-Hui Chen \\ Department of Materials Science and Engineering, National Cheng Kung University, Tainan, Taiwan 701, R. O. China
}

In this study, 5052 Al-Mg alloy was pre-treated and friction stir processing (FSP) was then performed on the specimens to explore the deformation resistance. The experimental results indicate that the FSP specimens not only had better tensile properties but also better vibration fracture resistance. It is significant that the tensile elongation and deformation resistance tended to increase as the grain was refined by friction stir processing. Results show that the use of FSP yielded uniform hardness distribution and slightly larger $\mathrm{n}$ values which improved the vibration fracture resistance of the specimens. However, even without FSP, more uniform elongation and larger $\mathrm{n}$ values could still improve the vibration fracture resistance of the specimens. Consequently, the uniform elongation and larger $\mathrm{n}$ values play an important role in increasing vibration fracture resistance. As for crack propagation, the FSP specimens revealed only a few slip bands in the vicinity of the main crack, and the $5052 \mathrm{H} 34$ specimen displayed a few slip bands in the vicinity of the main crack. However, more slip bands were observed in the vicinity of the main crack on the 5052-O specimens, and there were deformation traces and advancing cracks rising at the front of the main crack. Consequently, vibration fracture resistance can also be better improved if the crack initiation and propagation through the stir zone of FSP specimens are controlled. This is correlated with the formation of fine grains through dynamic recrystallization in the vicinity of stir zone.

(Received August 24, 2005; Accepted October 11, 2005; Published December 15, 2005)

Keywords: aluminum-magnesium alloy, vibration fracture resistance, dislocation, sub-boundaries, slip band

\section{Introduction}

Many different Industries now pay much attention to lightweight and fuel economic materials. 5xxx aluminum alloys which have excellent formability, good strength and high corrosion resistance are widely used in the automotive and shipbuilding industries. However, these alloys implemented in car bodies show a tendency towards an abnormal vibration process (a typical plate failure). Severe fracture problems occur especially when the applied vibration frequency meets the resonance. ${ }^{1)}$ Consequently, the resonant vibration fracture behavior needs to be thoroughly explored.

On the other hand, as described by the Hall-Petch relationship, grain refinement is quite effective in improving mechanical properties, such as hardness and strength. ${ }^{2,3)}$ Friction Stir Welding (FSW) is a potential technique of joining light metals. In the joining process, through severe plastic deformation, there will be fine recrystallized grains in the friction stir zone during welding. ${ }^{4-6)}$ Therefore, we focused our attention on this unique characteristic of FSW and have studied this process not as a welding technique but as a new grain refinement process. ${ }^{7,8)}$ Consequently, in this study, friction stir is only regarded as a new grain refinement process. Figure 1 shows a schematic illustration of the basic principle of friction stir processing (FSP).

However, previous reports have pointed out ${ }^{9-11)}$ the temperature in the vicinity of the FSW-tool generally shows an asymmetric distribution between the advancing side and the retreating side. It should be noted that even slight variations in elevated temperature can cause significant changes in the microstructure and mechanical properties. Consequently, in this study, in order to eliminate the influence of advancing or retreating microstructure, specimens were cut from the friction stir zone, as indicated in Fig. 2.

\footnotetext{
${ }^{* 1}$ Graduate Student, National Cheng-Kung University

${ }^{* 2}$ Corresponding author, E-mail: z7408020@email.ncku.edu.tw
}

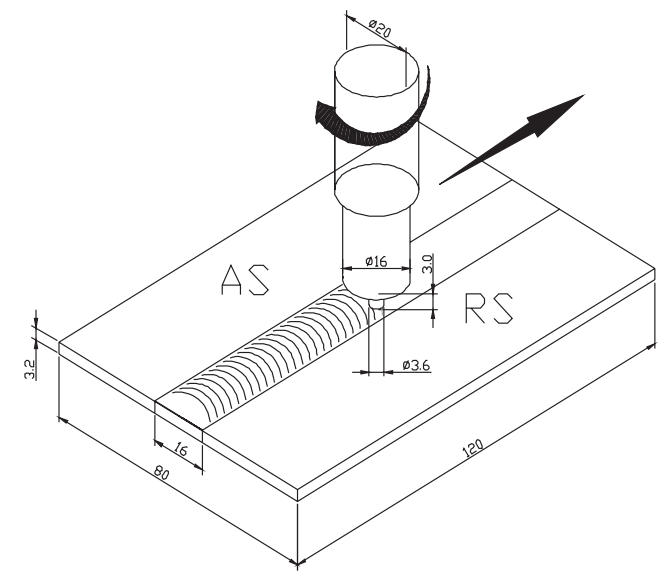

Fig. 1 Schematic illustration of the friction stir process (FSP).

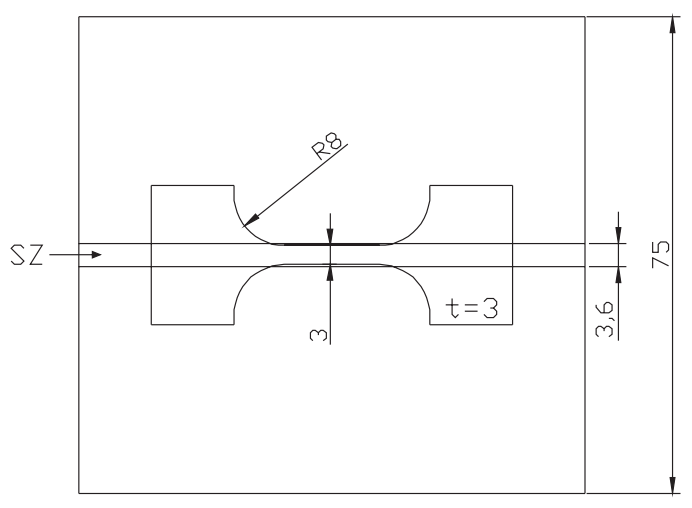

Fig. 2 Schematic illustration of the tensile specimen (FSP).

$5 x x x$ aluminum alloys have great potential for refining the microstructure through deformation process, allowing the mechanical properties of $\mathrm{Al}-\mathrm{Mg}$ alloys to be enhanced. ${ }^{12)}$ This study focuses on the formation of fine grains through dynamic recrystallization in the stir zone and will further- 
Table 1 Chemical composition of the samples (mass\%).

\begin{tabular}{ccccccc}
\hline & $\mathrm{Mg}$ & $\mathrm{Fe}$ & $\mathrm{Cr}$ & $\mathrm{Si}$ & $\mathrm{Mn}$ & $\mathrm{Al}$ \\
\hline 5052 & 2.58 & 0.26 & 0.21 & 0.15 & 0.1 & Bal. \\
\hline
\end{tabular}

more clarify the effect of microstructural evolution on vibration fracture behavior. The $5052 \mathrm{Al}-\mathrm{Mg}$ alloy was chosen to explore the damping capacity and the vibration fracture behavior under resonant vibration.

\section{Experimental Procedures}

5052H34 Al-Mg plates were chosen to achieve a fullyannealed microstructure at $625 \mathrm{~K}$ for $2 \mathrm{~h}$, after which the plate and fully-annealed plate were subjected to a single pass by FSP (tool rotation speeds were performed at $2300 \mathrm{~min}^{-1}$; tool moving speeds were fixed at $160 \mathrm{~mm} \mathrm{~min}^{-1}$ with $1.5^{\circ}$ of tool angle; the downward push force was controlled at 19.6 MPa). The chemical compositions of the plates listed in Table 1. The 5052H34 specimen and the fully-annealed specimen are designated as "H34" and "O" in this paper. The stirred specimens of H34 and $\mathrm{O}$ are designated as "FSP-H34" and "FSP-O", respectively.

Microstructural features of the specimens were examined by optical microscopy (OM), electron backscattered diffraction (EBSD) and transmission electron microscopy (TEM). The thin-foil disk specimens of HF2000 FE-TEM were cut $3 \mathrm{~mm}$ in diameter from the stir zone and were then jet electropolished by an electrical discharge machine in a $50 \mathrm{~mL}$ $\mathrm{HNO}_{3}+200 \mathrm{~mL} \mathrm{CH} \mathrm{CH}_{3} \mathrm{OH}$ solution at $248 \mathrm{~K}$. To study the Vickers hardness profile in the vicinity of the stir zone, the Vickers indenter was set to be a $0.98 \mathrm{Nf}$ load for $15 \mathrm{~s}$. In the tensile test, in order to examine the effects of the micro- structural features and tensile properties, the strain rate was fixed at $8.3 \times 10^{-4} \mathrm{~s}^{-1}$, and a tensile specimen with $15 \mathrm{~mm}$ gauge and $3 \mathrm{~mm}$ thickness was used. As indicated in Fig. 2, the FSP specimens were longitudinal to the stir zone.

The vibration specimen and its dimensions are illustrated in Fig. 3(a). To generate the vibration, one end of the test specimen was clamped on a vibration shaker. Figure 3(c) schematically depicts the vibration setup.

The two V notches adjacent to the clamp were designed to confine the resonance to the stir zone. Deflection of the specimen at the other end opposite the vibration shaker was measured using a deflection sensor. As shown in Fig. 3(b), in order to determine the resonance frequency which led to the largest deflection, the vibration frequency was varied continuously.

For both the $\mathrm{H} 34$ and $\mathrm{O}$ specimens, the resonant frequencies were in the range of $42.5 \pm 1 \mathrm{~Hz}$, and the resonant frequencies of the stirred specimens shifted from $42.5 \pm 1$ to $41.5 \pm 1 \mathrm{~Hz}$.

\section{Results}

\subsection{Microstructural feature and tensile properties}

Figure 4 shows the typical metallography of each specimen. As indicated in Figs. 4(a) and (b), the FSP-H34 and FSP-O specimens had a fine equiaxed grain structure in the stir zone. According to the average grain size shown in Table 2, there is only a slight difference between the microstructures of the stirred plates. The H34 has a pancake-like elongated structure and the $\mathrm{O}$ has an elongated coarse grain structure. The TEM images of these specimens are shown in Fig. 5. Figures 5(a) and (b) show that both FSPH34 and FSP-O demonstrated fine equiaxed grains with a low density of dislocation and sub-boundaries; the H34 consisting (a)

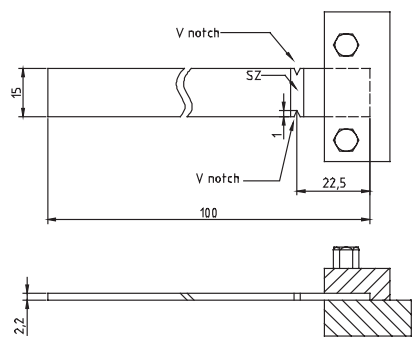

(c)

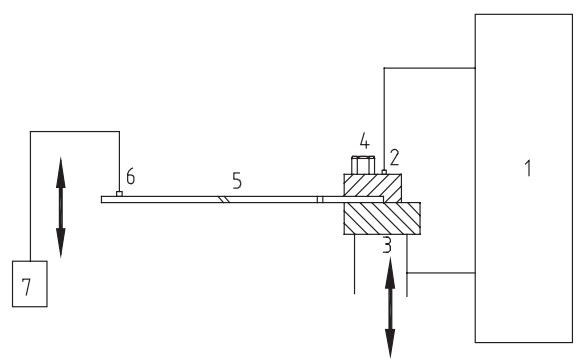

(b)

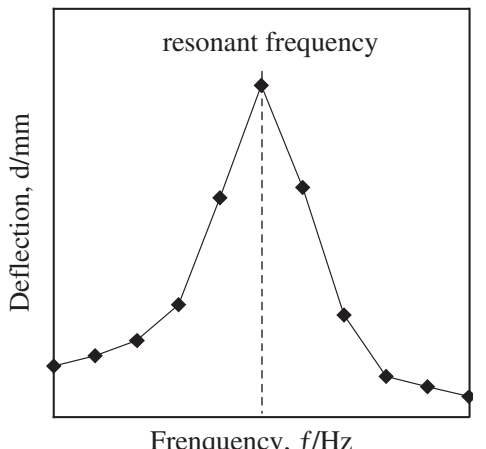

Frenquency, $f / \mathrm{Hz}$
1: vibration controller
5: specimen
2: acceleration sensor
6: deflection sensor
3: vibration shaker
7: recorder
4: specimen clamp

Fig. 3 Shape and dimension of the resonant vibration test specimen for (a) specimen geometry and dimension, (b) resonant frequency, (c) the resonant vibration setup. 
(a)
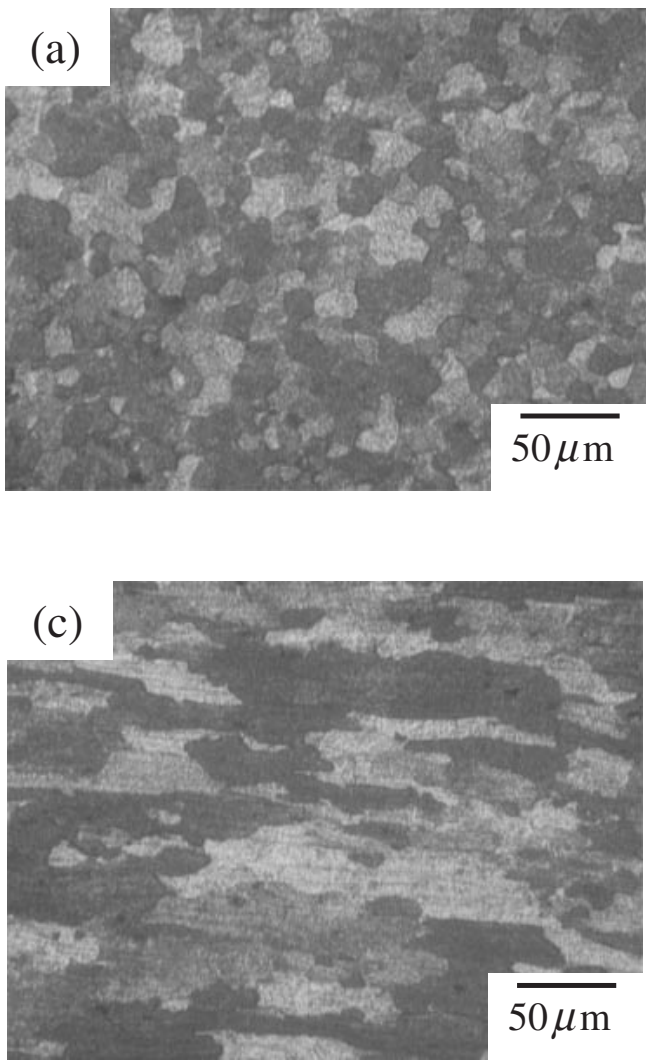
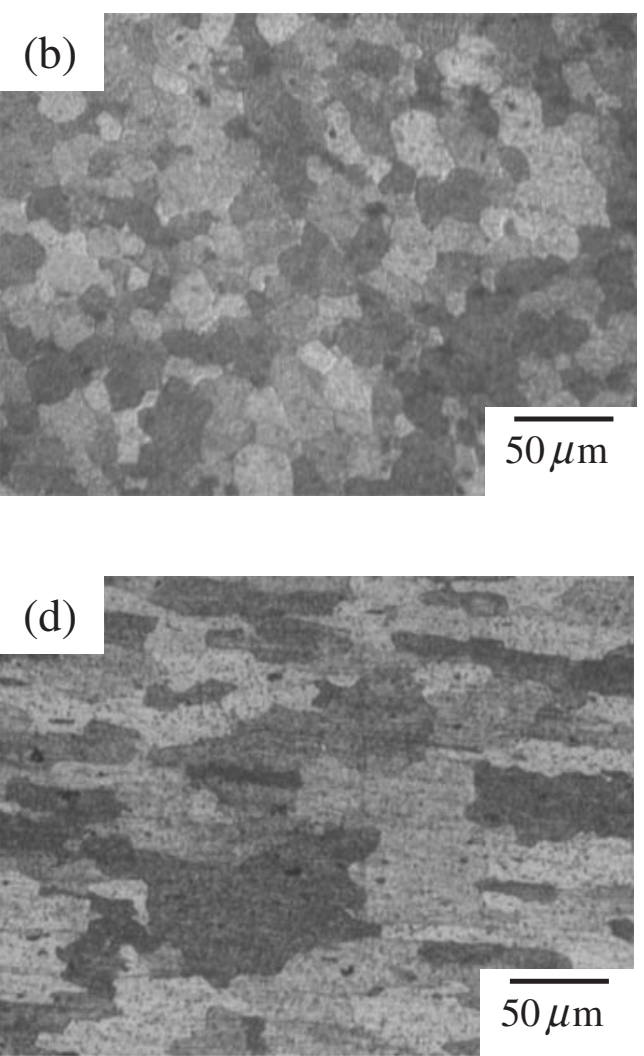

Fig. 4 Optical microscopic of (a) FSP-H34 (b) FSP-O (c) H34 (d) O.
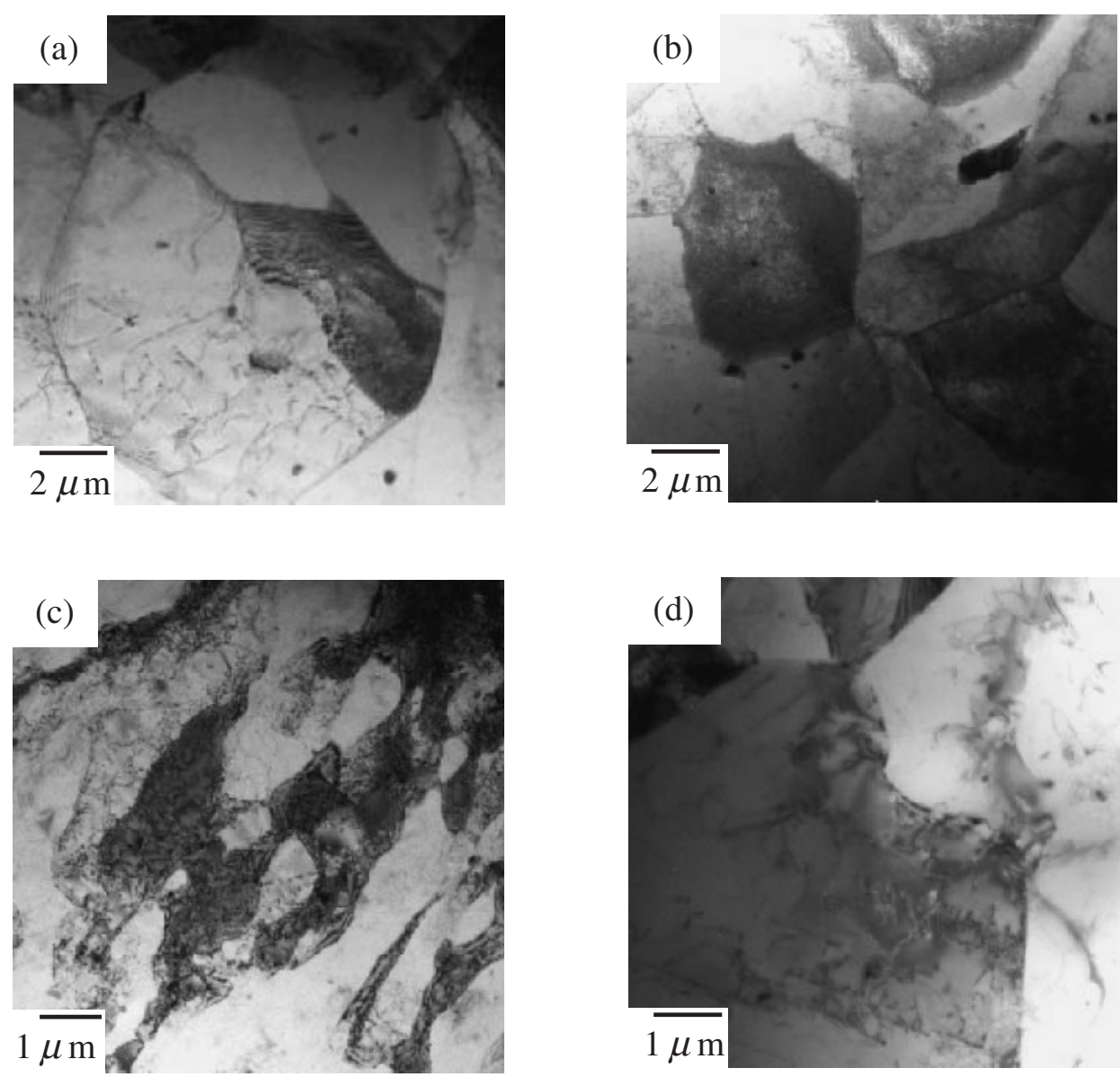

Fig. 5 TEM micrographs of (a) FSP-H34 (b) FSP-O (c) H34 (d) O. 
of elongated grains with a high density of dislocation tangles and sub-boundaries is shown in Fig. 5(c); the $\mathrm{O}$ specimen exhibiting coarse grains with a low density of dislocation and few sub-boundaries is shown in Fig. 5(d).

After annealing treatment, as shown in Fig. 6, the tensile data shows that the strength of the specimens has decreased and the elongation has increased. Based on the tensile data obtained from FSP-H34 and FSP-O which were cut entirely from the stir zone, also shown in Fig. 6, it is clear that the friction stirred specimens displayed an improvement in uniform elongation. This can be easily proven by comparing the results shown in Table 2 which reveals that grain refinement is very effective in enhancing elongation. In

Table 2 The average grain size, yield strength and $\mathrm{n}$ valves of the samples.

\begin{tabular}{lccrc}
\hline \multicolumn{1}{c}{ Specimen } & FSP-H34 & FSP-O & H34 & O \\
\hline Average grain size $(\mu \mathrm{m})$ & 13.4 & 14.1 & 42.3 & 46.6 \\
Yield strength $(0.2 \%)(\mathrm{MPa})$ & 105.4 & 104.2 & 166.0 & 100.7 \\
n values & 0.18 & 0.20 & 0.12 & 0.19 \\
\hline
\end{tabular}

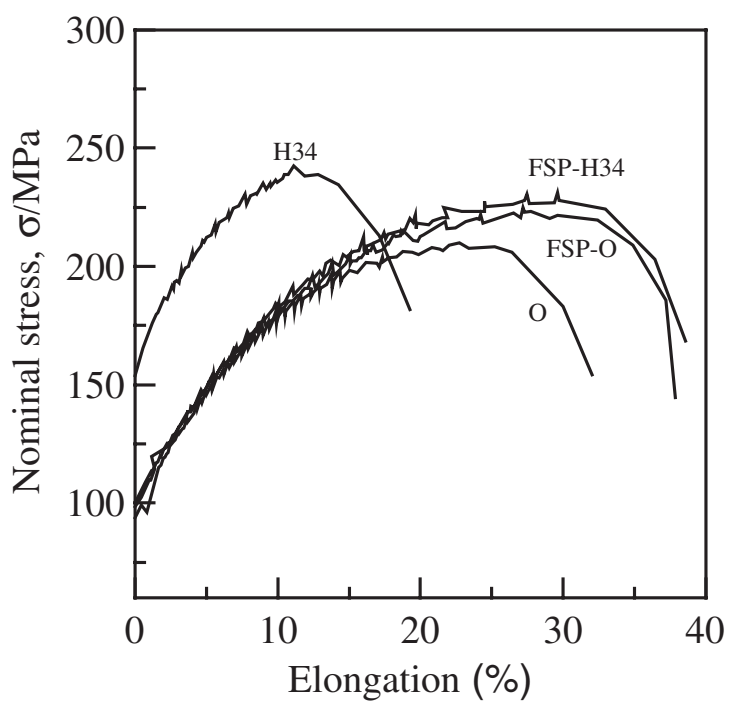

Fig. 6 Relation between stress and elongation.

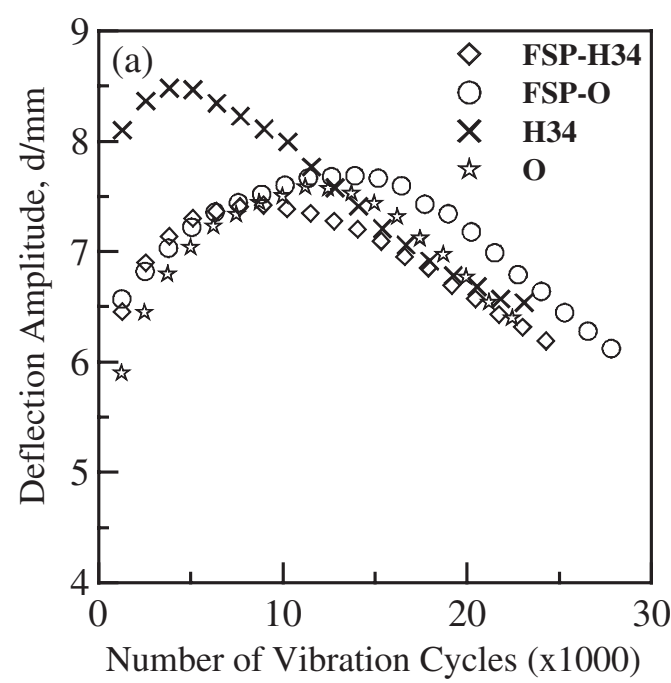

addition, FSP-H34 and FSP-O are not significantly different, even though their grain size and density of dislocation are slightly different. The microstructural feature of the stirred specimens shows that the tensile elongation tends to increase as the microstructure is refined in the friction stir processing, which is probably correlated with the phenomenon of dynamic recrystallization.

\subsection{Deflection and crack propagation features}

Figure 7 shows the vibration test results presented in the form of D-N curves (deflection amplitude vs. number of cycles). The D-N curve suggests that the deflection amplitude can be classified into two stages. Stage I: The deflection amplitude increases as vibration cycles increase until a maximum is reached. Stage II: The deflection decreases from the peak value as the vibration cycles increase further.

The D-N curves of all test specimens shown in Fig. 7(a) were recorded under an identical acceleration $(g=1.1)$. In Stage I, the initial ascending deflection is inversely proportional to the damping capacity, and as the damping capacity increases, the initial deflection amplitude drops. Furthermore, there is distinct difference in the duration of the period of ascending deflection amplitude among the FSP-H34, FSP-O, $\mathrm{H} 34$ and $\mathrm{O}$ samples. Consequently, the variation in the number of vibration cycles leads to the conclusion that the duration of the ascending deflection amplitude should be correlated with the vibration fracture resistance. Figure 7(b) demonstrates that these D-N curves display a similar feature when the specimens are tested under constant initial deflection amplitude.

As shown quantitatively in Fig. 8, the duration of stage I of the $\mathrm{D}-\mathrm{N}$ curve increases as the $\mathrm{n}$ values increase. It is reasonable to suggest that the strain hardening during the vibration test occurred in stage I. During the initial stage of ascending deflection, the microhardness at the vicinity of the main crack increased as the number of vibration cycles increases. Taking the FSP-O specimen for example, the original microhardness before vibration testing was 73.5 VHN. The microhardness increased to $83 \mathrm{VHN}$ at vibration cycles of 5000 (The FSP-O specimen, as indicated

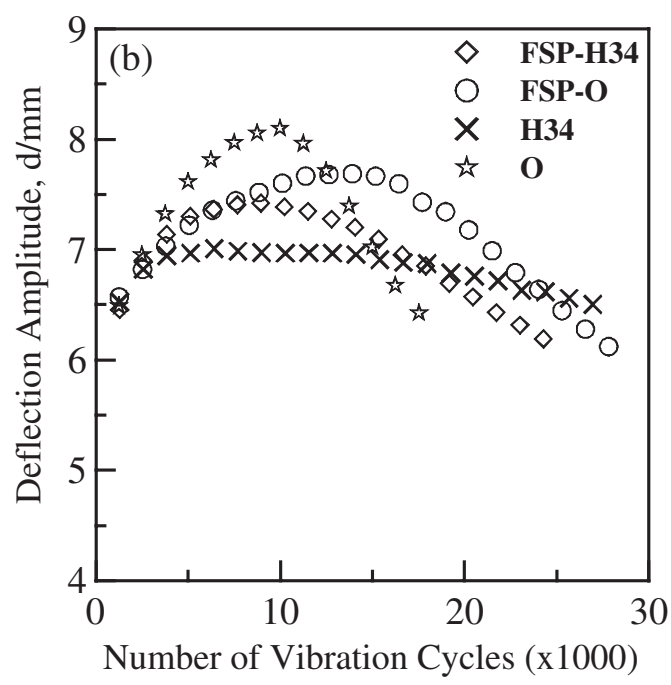

Fig. 7 Deflection $v s$. number of vibration cycles curve (a) $g=1.1$ (b) D $=6.5 \mathrm{~mm}$. 
in Fig. 7(b), is in the stage of ascending deflection). The final hardness reached $86.6 \mathrm{VHN}$ in the stage of maximum deflection.

Figure 7(b) also shows the D-N curves of the specimens with and without friction stir processing. As illustrated in Fig. 8, the D-N curves of the specimens with FSP reveal that the duration of stage I significantly increased, which corresponds to a significant increase of $\mathrm{n}$ values. This data will be further examined in the discussion section.

Observed from an optical microscope, as shown in Fig. 9, the crack propagation behavior of all the specimens was different. Figures 9(a) and (b) show that the few slip bands on the specimens with FSP are observed in the vicinity of the

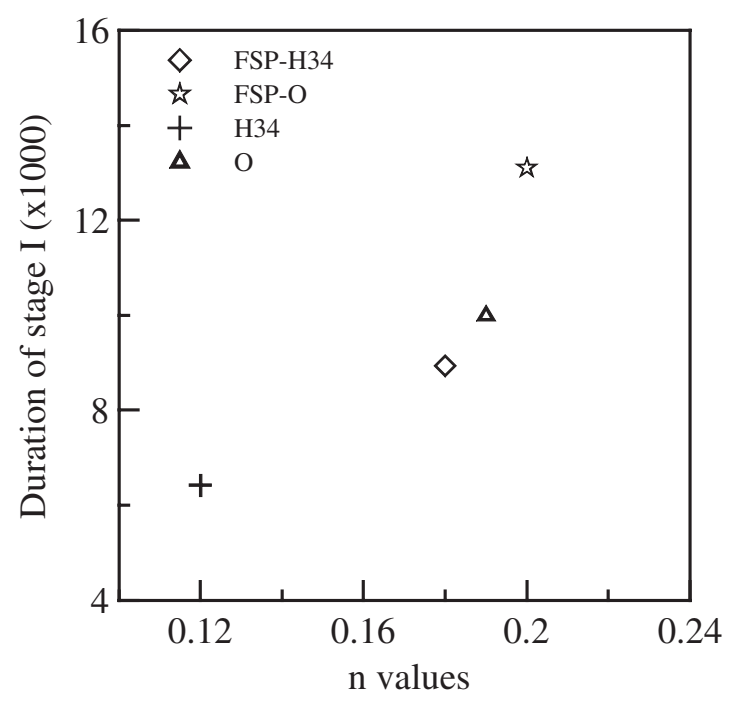

Fig. 8 Relation between $\mathrm{n}$ values and duration of stage I.
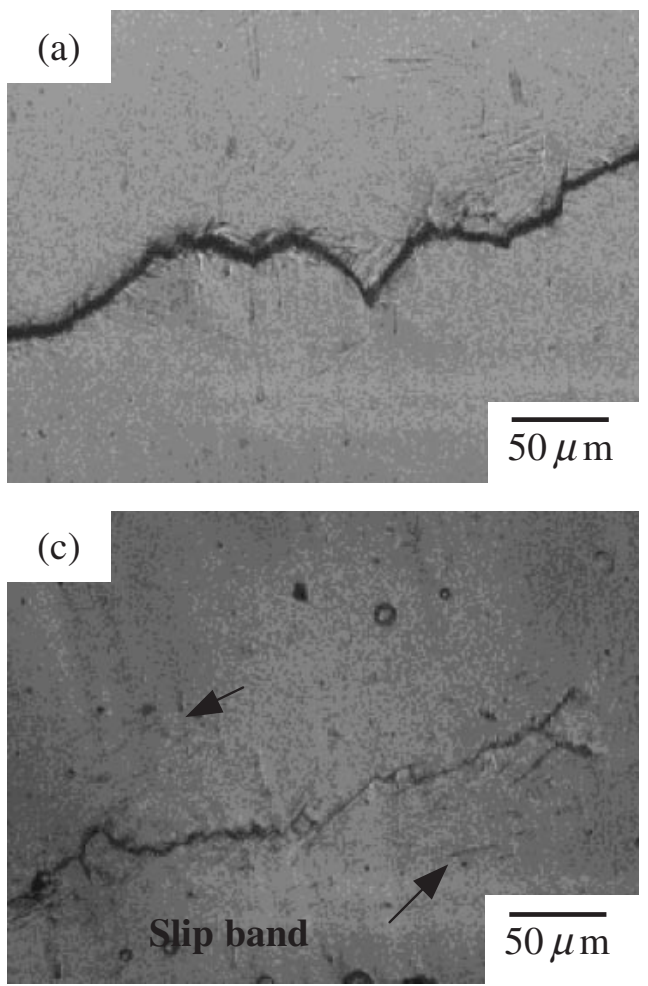

main crack throughout the testing period. In addition, in Fig. 9(c), there are a few slip bands observed in the vicinity of the main crack in the H34 specimen; in Fig. 9(d), there are more slip bands in the $\mathrm{O}$ specimen.

As illustrated in Figs. 7 and 9, the vibration fracture resistance of each specimen is associated with the microstructural feature. In Figs. 9(a) and (b) only a few slip bands in the vicinity of the stir zone are observed, and the SEM images are shown in Figs. 10(a) and (b). This is correlated with the formation of the fine grains through dynamic recrystallization in the vicinity of the stir zone. On the other hand, the H34 specimen showed still a few slip bands in the vicinity of the main crack when the deflection amplitude reached the maximum, as shown in Fig. 10(c). However, when the deflection amplitude reached the maximum, more slip bands appeared in the vicinity of the main crack in the $\mathrm{O}$ specimen, and deformation traces and advancing cracks rose at the front of the main crack, as seen from the SEM images in Fig. 10(d). The microstructural feature in the vicinity of the notches region can now be elucidated. Consequently, as shown in Figs. 9 and 10, the cracking features of the vibration specimens are related to the cracking propagation behavior and their vibration fracture resistance. A significant difference in the cracking propagation behavior of all the vibration specimens is recognized. Furthermore, from observation of the main cracks on the etching specimens of the stirred and unstirred samples, few differences are recognized, as illustrated in Fig. 11. However, for the unstirred specimens, a significant improvement in vibration fracture resistance is recognized in Table 3, which is correlated with the variation in crack tortuosity. For the stirred specimens, also shown in Table 3, very little difference is recognized in crack tortuosity values, nevertheless the duration of stage I
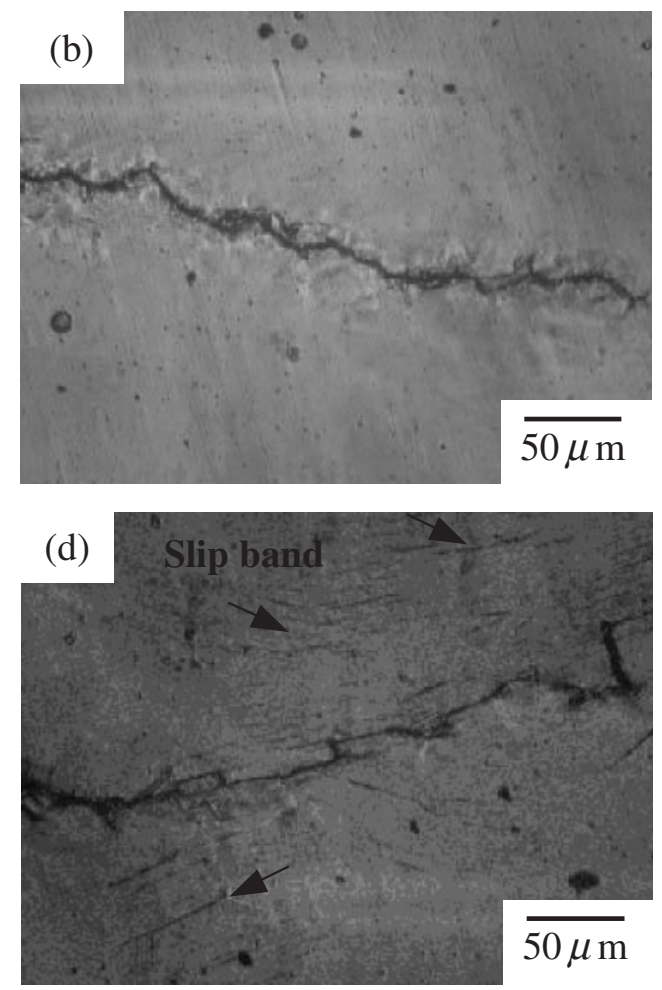

Fig. 9 Optical micrographs showing the macroscopic crack propagation (a) FSP-H34 (b) FSP-O (c) H34 (d) O. 

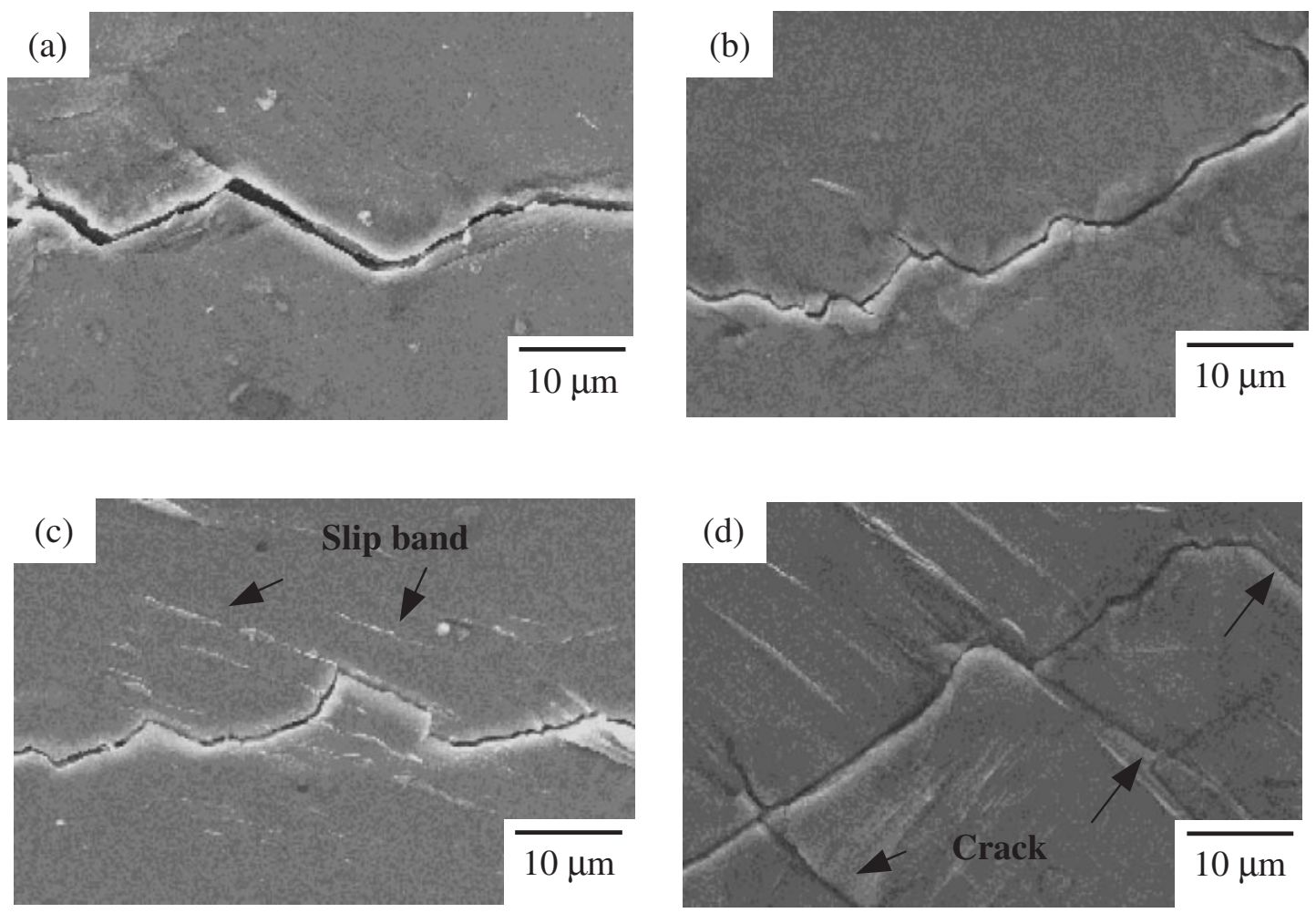

Fig. 10 SEM micrographs showing the crack propagation path of (a) FSP-H34 (b) FSP-O (c) H34 (d) O.
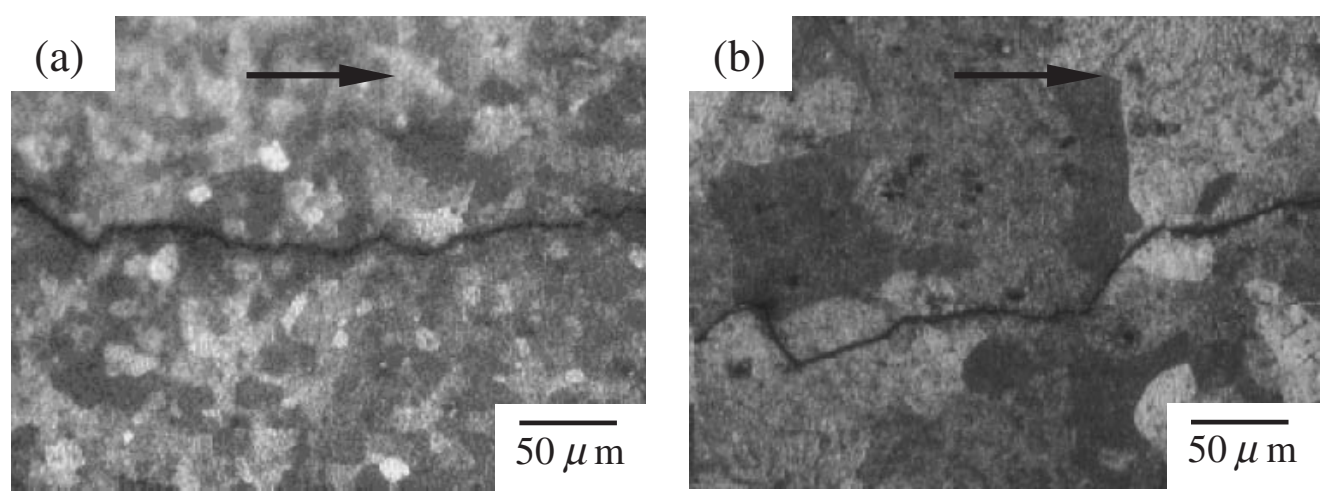

Fig. 11 Optical microstructure of (a) FSP-H34 (b) H34. The arrows indicate the macroscopic crack propagation direction.

Table 3 Crack path tortuosity in test specimens.

\begin{tabular}{ccccc}
\hline Specimen & FSP-H34 & FSP-O & H34 & O \\
\hline Crack path tortuosity & 1.15 & 1.16 & 1.18 & 1.21 \\
\hline $\begin{array}{c}\text { Duration of stage I }(\times 1000) \\
\text { (constant D) }\end{array}$ & 8.9 & 13.9 & 6.42 & 9.99 \\
\hline $\begin{array}{c}\text { Duration of stage I }(\times 1000) \\
\text { (constant G) }\end{array}$ & 8.9 & 13.9 & 3.8 & 11.2 \\
\hline
\end{tabular}

value represents a significant difference between the FSPH34 and FSP-O specimens.

\section{Discussion}

As annealing proceeds, the dislocation begins to form low energy tilt boundaries. When the structure is reasonably stable, the rate of dislocation annihilation is much slower.
Therefore, as shown in Fig. 5, two recovery mechanisms, dislocation annihilation and rearrangement, operate simultaneously into stable configurations. This illustrates that the dislocation density plays an important role in decreasing yield stress and increasing elongation. Moreover, some previous studies ${ }^{13,14)}$ have actually pointed out that such dynamic recrystallization often leaves some grains with a high density of dislocations in the stir zone, which suggests that the dislocation densities of the stir zone are higher than those of annealed materials but lower than those of strainhardened materials.

The stress-elongation curves in Fig. 6 show that all the tensile test specimens exhibited the serrated phenomenon, which is also known as the PLC effect (the solute-dislocation occurs mainly at the obstacles where dislocations are temporarily held. The solute atoms can then diffuse to these dislocations and cause atmospheres to form around them. When the deformation continues, the dislocations have to 
(a)

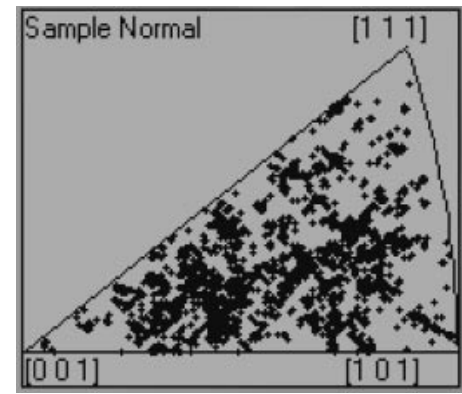

(b)

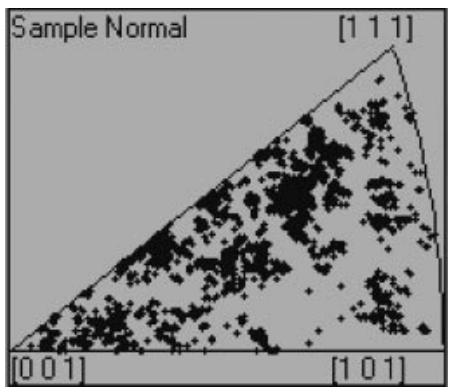

Fig. 12 The inverse pole figure of (a) FSP-H34 (b) FSP-O.

break away from these atmospheres and multiply. This leads to serrated yielding $\left.{ }^{15-17}\right)$. The higher densities of dislocation and grain boundary introduced by strain hardening can hold the mobile dislocations long enough to let $\mathrm{Mg}$ atoms form atmospheres around them. ${ }^{18)}$ Consequently, before necking of specimen occurs, the PLC effect increases as strainhardening increases. Moreover, the effect of PLC shown in the stress-elongation curve is more significant in the specimens which underwent FSP. This can be ascribed to the grain refinement and the resulting increase of the boundaries which is the main source of obstacles.

From the crack propagation behavior mentioned above, the feature of D-N curves (deflection amplitude vs. number of vibration cycles) can be generalized into two stages, as indicated in Figs. 7(a) and (b): working hardening and main crack formation. The increase of work hardening can raise the effective elastic modulus, and the deflection amplitude then increases because the damping capacity of the specimen drops. ${ }^{19)}$ This work hardening stage is designated as stage I, and the vibration fracture resistance is strongly dependent on the duration of stage I. As the number of vibration cycles increases, there is a drastic decrease in deflection amplitude, designated as stage II, which results from the deviation of the actual vibration frequency from the resonant frequency and the inward propagation of the major crack. ${ }^{20)}$

Both the FSP-H34 and FSP-O specimens display low dislocation densities and a random distributed texture, as shown in Fig. 12. Consequently, they have the same microstructure, which is related to dynamic recrystallization. There is also no significant difference between the microhardness in the stir zone of the FSP-H34 and FSP-O specimens, which is shown in Fig. 13. However, the FSP-H34 specimens exhibits inhomogeneous hardness distribution and slightly smaller $\mathrm{n}$ values, as shown in Table 2, which is associated with prestrain in the parent plate. Consequently, as illustrated in Table 3, the friction stirred specimens have slightly different tortuosity, which is related to vibration fracture resistance. For the FSP-H34 specimen, its inhomogeneous hardness and slightly smaller $\mathrm{n}$ values are attributable to the existence of prior strain hardening in the grains. Therefore, the FSP-O specimen has a longer period of deflection amplitude and greater vibration fracture resistance.

On the other hand, for the specimens which did not undergo FSP, the $\mathrm{O}$ specimen possesses not only better damping capacity but also a longer stage I duration, which is shown in Figs. 7(a) and (b). Both Figs. 4 and 13 show that there is a significant difference in the microstructure and

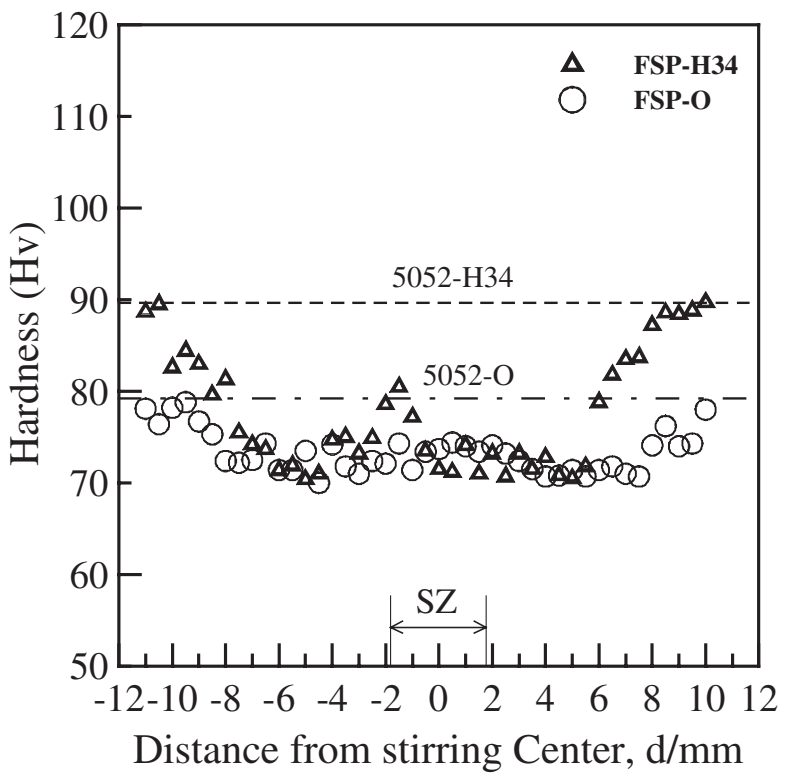

Fig. 13 Hardness profile across the stir zone.

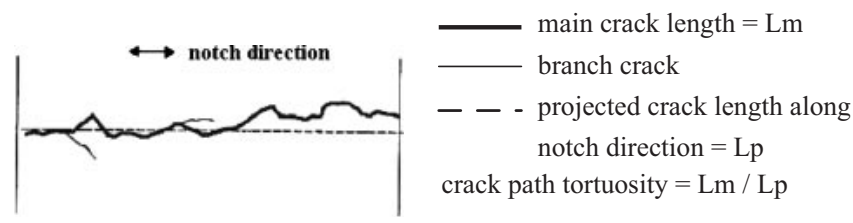

Fig. 14 Schematic drawing of the cracking features generated by resonant.

microhardness of the $\mathrm{H} 34$ and $\mathrm{O}$ specimens. This result can be further compared with their uniform elongation data (H34: $7.6 \%, \mathrm{O}: 15 \%)$ and $\mathrm{n}$ values. The duration of stage $\mathrm{I}$ for the $\mathrm{O}$ specimen, which has larger uniform elongation and $\mathrm{n}$ values, is also longer. Consequently, the $\mathrm{O}$ specimen has better work hardening behavior. This is attributable to its microstrauctural characteristic of annealing treatment which improves the vibration fracture resistance.

The effect of microstructural differences on crack propagation paths was examined by an optical microscope equipped with an image analyzer. All specimens were polished on the surface with $0.3 \mu \mathrm{m} \mathrm{Al}_{2} \mathrm{O}_{3}$, so the cracking path through the microstructure could be directly observed. Consequently, as shown in Fig. 14, quantitative data of crack tortuosity could then be then determined: the crack tortuosity value equals the main crack length divided by the projected 
crack length along the notch direction (the projected crack length is designated as the dotted line in Fig. 14). The projected crack length is parallel to the tip direction of the two $\mathrm{V}$-notches [i.e., the notch on the stir zone is indicated in Fig. 3(a)]. In this study, the tortuosity of the main crack for all the specimens was calculated (Table 3 ).

As for the vibration fracture resistance, the crack propagation behavior is influenced by microstructural variation. Figures 9 and 10 reveal that there are significant differences in the crack propagation behavior between the specimens which had and did not have FSP performed. Compare the result with the D-N curve shown in Fig. 7. The specimens with FSP possess better vibration fracture resistance because of the microstructural refinement and a slight increase in $n$ values, while on the specimens without FSP, there are more slip bands introduced by vibration testing which enhanced the ability to absorb vibration energy. Consequently, the $\mathrm{O}$ specimens possess better vibration fracture resistance. Based on the microstructural characteristics of all the vibration specimens, it is clear that the grain refinement and $n$ values play an important role in the crack propagation behavior. These results agree well with the variation in crack tortuosity which is indicated in Table 3. Although the effect of the stirred specimens on the microstructural refinement is recognized, the existence of pre-strain on specimens which underwent FSP needs to be further examined.

\section{Conclusions}

According to the experimental results discussed in the previous sections, the following conclusions can be drawn:

(1) Annealing treatment is effective in improving the damping capacity and is also able to enhance the vibration fracture resistance of $\mathrm{Al}-\mathrm{Mg}$ alloy under resonant vibration.

(2) After FSP, the microstructures of the specimens acquire grain refinement, low dislocation densities and a random distributed texture.

(3) The tensile deformation resistance and uniform elongation increases after FSP is performed. In particular, the elongation can be significantly improved after the microstructure is refined during dynamic recrystallization.

(4) The vibration fracture resistance of friction stirred specimens is closely related to the duration of stage I. The microhardness in the stir zone of FSP-O is more uniformly distributed, and its slightly larger $\mathrm{n}$ values have an influence on prolonging the vibration fracture resistance. The vibration fracture resistance of specimens with or without FSP is quantitatively correlated with the crack tortuosity value which corresponds to the crack propagation behavior.

(5) For the specimens which did not undergo FSP, more slip bands introduced by vibration testing enhanced the ability to absorb the vibration energy and consequently improved the crack propagation resistance.

\section{Acknowledgement}

Financial support from the National Science Council of Taiwan is gratefully acknowledged (contract No. NSC 942216-E-006-034).

\section{REFERENCES}

1) K. T. Huang, T. S. Lui and L. H. Chen: Mater. Trans. 45 (2004) 32163222.

2) Y. J. Kwon, I. Shigematsu and N. Saito: Mater. Trans. 45 (2004) 23042311.

3) Y. S. Sato, M. Urata, H. Kokawa and K. Ikeda: 6th International Trends in Welding Research Conference Proceedings, (2002) pp. 15-19.

4) C. G. Rhodes, M. W. Mahoney, W. H. Bingel, R. A. Spurling and C. C. Bampton: Scr. Mater. 36 (1997) 69-75.

5) G. Liu, L. E. Murr, C. S. Niou, J. C. McClure and F. R. Vega: Scr. Mater. 37 (1997) 355-361.

6) Y. S. Sato, M. Urata, H. Kokawa and K. Ikeda: Mater. Sci. Eng. A 354 (2003) 298-305.

7) Y. J. Kwon, I. Shigematsu and N. Saito: Mater. Trans. 44 (2003) 13431350 .

8) I. Charit and R. S. Mishra: J. Mater. Res. 19 (2004) 3329-3342.

9) M. Maeda, H. Liu, H. Fujii and T. Shibayanagi: Welding in the world 49 (2005) No. 3/4 69-75.

10) H. Lui, M. Maeda, H. Fujii and K. Nogi: J. Mater. Sci. Lett. 22 (2003) 41-43.

11) H. Lui, H. Fujii, M. Maeda and K. Nogi: J. Mater. Sci. Lett. 22 (2003) 1061-1063.

12) Y. S. Sato, H. Kokawa, K. Ikeda, M. Enomoto, S. Jogan and T. Hashimoto: Metall. Mater. Trans. A 32 (2001) 941-948.

13) K. V. Jata and S. L. Semiatin: Scr. Mater. 43 (2001) 743-749.

14) Y. S. Sato and H. Kokawa: Metall. Mater. Trans. A 32A (2001) 30233031.

15) A. H. Cottrell: Philos Mag. 44 (1953) 829.

16) R. K. Ham and D. Jaffrey: Philos Mag. 15 (1967) 247.

17) M. C. Chen, L. H. Chen and T. S. Lui: Metall. Mater. Trans. 27A (1996) 1691-1694.

18) W. Wen and J. G. Morris: Mater. Sci. Eng. A 354 (2003) 279-285.

19) D. S. Jiang, T. S. Lui and L. H. Chen: Scr. Mater. 36 (1997) 15-20.

20) D. S. Jiang, T. S. Lui and L. H. Chen: Mater. Trans. 41 (2000) 499-506. 